\title{
The Effects of Frying Oils Supplemented with Vitamin E on Blood Parameters and Growth Performance of Rats
}

\author{
Fayez S. Hamam*, Ahmed S. Eldalo \\ Department of Pharmacology and Toxicology, College of Pharmacy, Taif University, Ta'if, Saudi Arabia \\ Email: ^f.hamam@tu.edu.sa
}

How to cite this paper: Hamam, F.S. and Eldalo, A.S. (2018) The Effects of Frying Oils Supplemented with Vitamin E on Blood Parameters and Growth Performance of Rats. Food and Nutrition Sciences, 9, 956-968.

https://doi.org/10.4236/fns.2018.98070

Received: June 29, 2018

Accepted: August 10, 2018

Published: August 13, 2018

Copyright $\odot 2018$ by authors and Scientific Research Publishing Inc. This work is licensed under the Creative Commons Attribution International License (CC BY 4.0).

http://creativecommons.org/licenses/by/4.0/

\section{(c) (i) Open Access}

\begin{abstract}
This study aimed at investigating effects of frying oils supplemented with vitamin E on blood parameters and growth performance of rats. Three experimental diets containing fresh corn oil or frying oil (FO) with or without vitamin E were fed to Wistar rats over a 90-day. Blood Chemistry, blood parameters (hematocrit and erythrocyte osmotic fragility) and growth performance were determined. Neither FO alone nor vitamin E had any considerable impact on the growth performance. Hematocrit of FO group did not change significantly compared to animals fed fresh oil. Although, vitamin E increased hematocrit to $16.37 \%$, but still it had no considerable effect on restoring hematocrit to normal values. Serum triacylglycerol (TAG) did not change significantly among FO animals, while significant increase in TAG was noted between FO and vitamin E treated animals compared with other treatments. Serum total cholesterol (TC) did not change significantly among experimental animals compared with the control. Vitamin E resulted in an insignificant increase in high-density lipoprotein (HDL-C) level compared to the control. Serum low-density lipoprotein (LDL-C) level did not display any considerable difference among the three treatments. Frying oil with or without vitamin E had no significant effects on growth performance, lipid chemistry, and blood parameters. The only significant effect of FO was shown on erythrocytes hemolysis at higher concentration. Vitamin E supplementation had no impacts on undesired effects caused by frying oil.
\end{abstract}

\section{Keywords}

Frying Oils, Vitamin E, Blood Parameters, Growth Performance

\section{Introduction}

Deep oil frying is a conventional and well-liked cooking tool used all over the 
world since its convenience as well as unique impacts on food flavour and quality. Thermally oxidized oil may include more than 400 different heat-stimulated reaction compounds, most of them transferred to the fried diet [1]. Owing to the possible toxicity of the oxidation compounds, these might cause a health danger to the public. Recently, the contribution of dietary oxidized lipids to the total energy uptake has increased in many countries, including KSA, mostly owing to the increasing fast food consumption that contained heated as well as processed dietary lipids. The people of KSA has experienced considerable alterations in their lifestyles, possibly due to several reasons, including fast socio-economic alterations, rapid urbanization, extensive reliance on cars, and increased dependence on telecommunication and computers. These factors have considerable impacts on lifestyle as well as eating habits among the society of KSA, as society drifts more and more towards "Western food" [2]. The prevalence of obesity, overweight, and dyslipidemia is on significant rise in the KSA as the numbers are very alarming to those who the health workers and to the public [2] [3] [4]. Several studies have demonstrated that thermally oxidized lipid impacts the growth performance of rats [1] and affects the serum lipoproteins profiles [5], serum antioxidant endogenous enzymes [5] [6], blood parameters (erythrocyte osmotic fragility and hematocrit \%) [7], blood pressure [8], level of thiobarbituric acid-reactive substances (TBARS) [5] [6], serum fatty acid profile [5] [6] [7], liver lipids [1] [6], rate of desaturation of fatty acids [1], and tocopherol level in theplasma [1] [6]. For example, Ammouche et al. [6] showed that oxidized sunflower oil had a toxic effect in animals, as reflected in higher level of malonaldehyde and a disturbance in antioxidant enzymes activities. Yen et al. [8] found that deep-frying oil decreased feed efficiency, while increased liver and kidney weights, and TBARS, in the meantime, feed efficiency and total antioxidant capacity were decreased in rats fed deep-frying oil (DFO). Additionally, DFO had no effect on blood pressure. Eder [1] showed that body weight (BW), feed conversion rates, desaturation rate of essential fatty acids (linoleic and $\alpha$-linolenic acids), liver and plasma tocopherols levels decreased among rats fed thermally oxidized lipid compared to those received the fresh oil. Zhong et al. [7] found that feeding juvenile fish (Atlantic cod Gadusmorhus) dietary oxidized lipid had no significant impacts on tested parameters, including growth performance, feed consumption, feed efficiency, hepatosomatic index, hematocrit and erythrocyte osmotic fragility. Nonetheless, this study suggested that supplementation of adequate amount of vitamin E could modifyundesiredeffects of oxidized oil in certain tissues. Olivero-David et al. [9] showed that total oxidized fatty acids and specific fatty acids (ketolinoleic) were effectively absorbed in the gastrointestinal tract of rats during the first $5 \mathrm{~h}$ post administration, but weakly absorbed in the case of fresh unoxidized oils. Oil alteration affected the digestibility of these fatty acids more than fasting, even though the digestibility of oxidized lipid was considerably influenced by fasting. Aladedunye and Przybyski [10] demonstrated that frying temperature and duration increased considerably 
$(\mathrm{P}<0.05)$ total polar components, $\mathrm{P}$-anisidine value, color and trans fatty acid (FA) content. In the meantime, the polyunsaturated FA (PUFA) content of frying oil decreased by half and the trans isomers increased by 2.5 folds during frying. Shafaeizadeh et al. [5] studied effects of uptake of oxidized oil with fibre supplementation on lipid profiles using animal model. It found that consuming fibre (pectin) could lower serum malondiaaldehyde and total cholesterol in rats fed oxidized oil. Many studies have been conducted to evaluate a range of chemical reactions and degree of oxidative deterioration by temperature of frying, several of the published data were attained via heating an oil in a laboratory setting but not during real fryingconditions. In the meantime, it has been noted that the chemical reactions that occur during deep-oil frying are unlike from those during heating [1]. In addition, different fats or oils behave differently concerning the rate of creation of polar components as well as secondary peroxidation products [1]. In recent times, much attention has been directed toward the biological impacts of oxidized fats or oils, and there is growing increasing evidences that oxidized lipids might be harmful to health, particularly in relation with atherosclerosis development, liver injury, and intestinal tumors promotion [1]. For example, Sebastian et al. [11] investigated quality and safety of deep-frying oils collected randomly from twenty different food outlets in Toronto, Canada, and found that all in-use as well as discarded oils demonstrated tremendously high amounts of oxidation products based on many methods used for assessing oxidative state of oil samples, namely the $\mathrm{P}$-anisidine value (p-AV) $(7.6<\mathrm{p}-\mathrm{AV}<56.5)$, elevated peroxide values $(\mathrm{PVs})(\mathrm{PV}>10 \mathrm{meq} / \mathrm{kg})$ and free fatty acid (FFA) levels (FFA $>1 \%$ ), suggesting that these restaurants used highly oxidized and hydrolyzed lipids in their normal commercial actions. Thus, owing to the probable toxicity of oxidation compounds, this might pose aserious health risk to the consumers. However, total polar compounds (TPCs), the main broadly used method of deep-frying oil quality in European countries, proposing that the tested oil samples were in the desired range $(15.5>$ TPC). Olivera-David [9] found that, in rats fed highly oxidized oil, the extended presence of high levels of oxidized fatty acids could result in damaging the intestinal tissues. If this hypothesis holds true, then feeding rats frying oil would affect growth performance, blood parameters, and blood lipid chemistry. Furthermore, vitamin $\mathrm{E}$ supplementation to animals received highly oxidized corn oil would alleviate undesired effects of such oil. To the best of our knowledge, this is the first intervention study in KSA used deep-frying oil collected from several restaurants in downtown Taif, KSA. The present study used in-use and discarded oil samples utilized in commercial activities and consumed on regular basis by the local people lived in Taif, KSA2.

\section{Materials and Methods}

\subsection{Collection of Oil Samples}

In the present study, fresh corn oil, in-use frying oil samples (turned into oxi- 
dized form through repeated heating at least 8 hours per day for three weeks on average) (250 $\mathrm{mL}$ per each restaurant) were obtained from ten randomly chosen restaurants in down town Taif, KSA. The oil in-use was stirred by means of a stainless steel spoon in order to attain homogenous samples, and then transferred to a dark colored bottle. The collected samples were filtered via a filter paper and kept at $4^{\circ} \mathrm{C}-6^{\circ} \mathrm{C}$ to avoid further oxidation and/or degradation.

\subsection{Feeding Study}

A total of 18 Wistar male rats were purchased from King Abdul Aziz university animal house (Jeddah, KSA). Rats were left for two weeks to acclimatize following transportation. Rats were housed at $25^{\circ} \mathrm{C} \pm 3^{\circ} \mathrm{C}, 12 \mathrm{~h}$ alternating light/ dark cycle, and a relative humidity was adjusted to $60 \% \pm 5 \%$. Eighteen rats aged $7-8$ weeks, weighing between 200 to $250 \mathrm{~g}$ were randomly allocated into three groups; each group made up of six animals. A control group consisting of animals fed a standard animal feed containing $10 \%$ fresh corn oil. The second group fed a diet containing $10 \%$ of frying oil without vitamin E supplementation. The third group composed of animals fed a standard diet with $10 \%$ frying oil and supplemented with vitamin E $(135 \mathrm{mg} / \mathrm{kg})$ (Sigma-Aldrich, Steinheim, Germany). Animals were allowed free access to feed and water throughout the 90 days feeding trial. Two animals were housed in a separate cage and kept in a room with a temperature at $23^{\circ} \mathrm{C}-25^{\circ} \mathrm{C}$. This was done in order to determine the amount of feed consumed every week. If each animal was housed in a separate cage, it would be more accurate to determine the amount of feed consumed, but because of limited number of cages, two rats were housed in each cage. Each animal was weight every week. Approximately $243-245 \mathrm{~g}$ of feed containing $10 \%(\mathrm{w} / \mathrm{w})$ fresh or frying oil was given to each cage for a period of seven days. The third group was given the same amount of feed containing $10 \%$ frying oil but supplemented with vitamin $\mathrm{E}$ ( $135 \mathrm{mg} / \mathrm{Kg}$ body mass). At the end of the seventh day, the remaining feed was removed, weight and the amount of food consumed was obtained by subtracting initial feed weight from feed weight at the end of the seven days feeding period. The remaining feed was discarded.

In vivo study of the present work received ethical approval from the Medical Ethical Committee at the Pharmacology and Toxicology Department, college of Pharmacy, Taif University (2017/tu/Pharmacy/05) and adhered to the ethical guidelines of EEC999 (European Union, 1982).

At the end of the study period (90 days), all animals were deprived from food at least 14 hours and in the morning scarified under chloroform. Blood withdrawing from retro-orbital vein was performed. Blood samples were collected into two tubes (with or without sodium citrate or ethylenediaaminetetra-acetic acid). Each animal was dissected and the livers were removed, washed with cold phosphate buffered saline (PBS), weight and stored at $-20^{\circ} \mathrm{C}$ for further analysis.

Hematocrit was measured via centrifuging the blood sample at $14,000 \mathrm{rpm}$ for 5 minutes using a Micro Hematocrit Centrifuge (Centurion Scientific, UK). Digital Hematocrit Reader (Iris International Inc., Westwood, MA, USA) was used 
to determine hematocrit percent. Hemolysis of erythrocytes (RBC) in PBS with different concentrations was used as an indicator of erythrocyte osmotic fragility (EOF). In brief, PBS was prepared and diluted to a serial dilution ranged from $0.11 \%$ to $0.85 \%$. Thirty microliters $(30 \mu \mathrm{l})$ of the whole blood was added to each concentration, shacked gently and left at room temperature for 30 minutes. After incubation, the blood samples were centrifuged at $750 \mathrm{rpm}$ for 5 minutes. The supernatant was removed and its absorbance was measured at $545 \mathrm{~nm}$ using UV-visible spectroscopy (UV-1800, Shimadzu, Kyoto, Japan). Determination of serum triacylglycerols, total cholesterol, and high-density lipoprotein (HDL-C), was performed spectrophotometrically using standard kits (Elascience, Wuhan, China), while level of low density lipoprotein (LDL-C) was calculated through applying the Friedwald's equation [12].

\subsection{Statistical Analysis}

Analysis of variance (one-way) was employed to compare among the treatments. Because there was a considerable difference, then the Tukey post hoc statistical test was employed to make comparisons (pairwise) between the three groups to determine where the difference lies. All data analysis was conducted using SPSS 22.0 (IBM-SPSS, Armonk, NY, USA). The significance of the differences among treatments was defined when $\mathrm{P}$ value $<0.05$. The resulted data were presented as the average (mean) \pm standard deviation $(n=6)$.

\section{Results and Discussion}

This is the first intervention research work conducted in KSA that used frying oil obtained from different local restaurants in downtown Taif, KSA. The present study used in-use and discarded oil samples utilized in commercial activities and consumed on regular basis by the local people lived in Taif, KSA. Table 1 showed growth performance of animals fed fresh corn oil, deep-frying oil with and without vitamin E supplementation. Neither oxidized oil alone nor oxidized oil with vitamin E supplementation had any considerable impact on the growth performance, namely final body weight, feed intake per each animal, feed efficiency and relative liver weight. Theoretically, feeding dietary oxidized oil could result in a decrease in body weight and, thus, decreases feed efficiency. Furthermore, when the antioxidants were added to the diet, it showed no significant impact on animals fed oxidized oil compared to those treated with fresh oil. For example, animals fed oxidized oil without supplementation showed a decrease in final body weight compared to those fed fresh corn oil, but this decrease was marginal in terms of statistics ( $266.81 \pm 41.89$ vs. $263.62 \pm 60.14)$. Animals fed frying oil without vitamin $\mathrm{E}$ supplementation showed a decrease in their final body weight compared to those fed fresh corn oil, but this decrease was insignificant, probably due to decreasing lipid digestibility and absorption. Another possible explanation of reduced weight could be related to reduced reactivity of pancreatic lipase in hydrolyzing thermally oxidized oils compared to the control 
group. However, this suggestion should be further investigated. Additionally, Wistar rats might adapt to accept thermally oxidized oil, at least in the short-range. If this feeding trial extended for a longer period, then the effects of oxidized oil on different variables of growth performance would be clearer. Our results agreed with those of Yen et al. [8] who found that feeding animals deep-frying oil resulted in a decrease in body weight and feed efficiency, while liver weight of rats received deep-frying oil was significantly higher than rats fed fresh oil. Despite increase in final body weight among animals fed oxidized oil and supplemented with vitamin E, but this increase was considered insignificant. Our results agreed with those of other relevant studies [1] [8]. For example, Yen et al. [8] found that feeding animals deep-frying oil resulted in a decrease in body weight and feed efficiency, while liver weight of rats received deep-frying oil was significantly higher than rats fed fresh oil. Although, results presented here suggested that vitamin E supplementation increases final body weight and feed intake compared to animals fed fresh and/or oxidized oils (Table 1), but this change was insignificant, when comparing it to the control group (266.81 \pm 41.89 vs. $284.87 \pm 38.78$ ) (Table 1). Similarly, Eder [1] found body mass gains and feed intake were slightly lower among rats given oxidized oil compared to their counterparts in the control group, liver weight was slightly increased in Eder's study which disagreed with findings of the present study. On contrary, Shafaeizadeh et al. [5] found that dietary oxidized oil did not result in liver enlargement.

The hematocrit of Wistar rats fed different diets is shown in Figure 1. On day 90 post treatment, hematocrit $(15.42 \%)$ of animals fed oxidized oil was lower than those received fresh oil (control), but this difference was insignificant. Although, vitamin E supplementation increased hematocrit percent to 16.37, but still supplementation had no considerable effect on hematocrit percent compared with the control animals. Hematocrit percent and erythrocyte osmotic fragility (EOF) are considered valuable indicators for chemical stress (e.g. oxidative stress) and environmental stress. Erythrocytes are one site of the main free radical production sites, which could stimulate oxidation reactions of polyunsaturated fatty acids among their phospholipids components of membranes and, hence change hematocrit level. Frying oil with or without supplementation had little effects on hematocrit, which disagreed with the findings of Mesembe et al. [13], who found that the packed cell volume of the rats fed thermoxidized palm oil was considerably lower than their counterparts in the control groups. The present study also disagreed with the findings of Ani et al. [14] who found that packed cell volume of Albino Wistar rats in the thermally oxidized palm oil fed group was considerably lower than their counterparts in the control group fed with the fresh palm oil. Zhong et al. reported similar results [7] but in juvenile Atlantic cod Gadusmorhus.

As presented in Figure 2, EOF determined as hemolysis of erythrocytes in normal saline solution with different concentrations. Erythrocytes hemolysis of animals in the three dietary treatments declined as the concentration of saline 


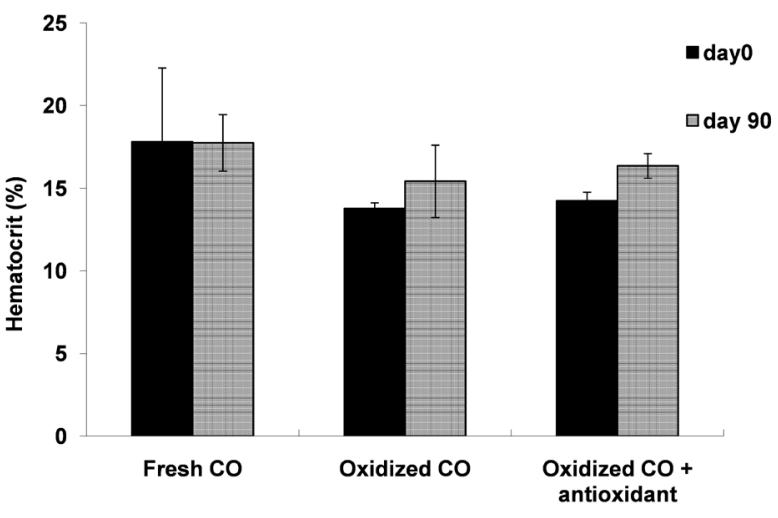

Figure 1. Hematocrit values obtained at day zero and day 90 of rats fed with oxidized corn oil (OCO) without antioxidants and with antioxidants (OCOA).

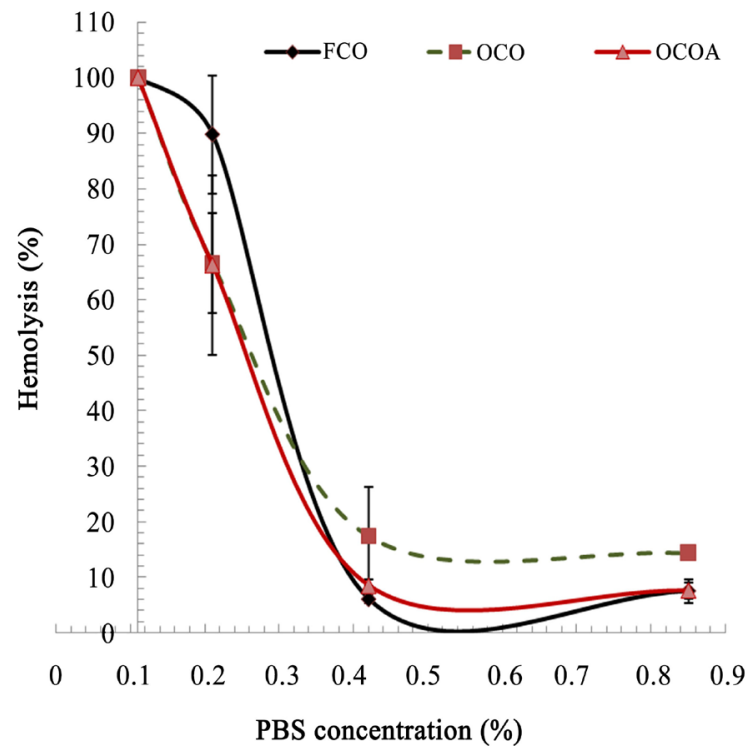

Figure 2. Effects of different diets on hemolysis (\%) in varying PBS concentrations. FCO stands for fresh corn oil, oxidized corn oil (OCO) without antioxidants and with antioxidants (OCOA).

Table 1. Growth variables in male Wistar rats fed fresh non-oxidized corn oil and deep-frying corn with and without antioxidant (vitamin E) over a 90-day feeding trial.

\begin{tabular}{cccc}
\hline Growth parameters & $\begin{array}{c}\text { Fresh corn oil } \\
(\text { FCO })\end{array}$ & $\begin{array}{c}\text { Deep-frying oil or oxidized } \\
(\text { OCO })\end{array}$ & $\begin{array}{c}\text { Oxidized corn oil with } \\
\text { vitamin E (OCOA) }\end{array}$ \\
\hline Initial weight $(\mathrm{g})$ & $253.86 \pm 15.86^{\mathrm{a}}$ & $257.04 \pm 23.23^{\mathrm{a}}$ & $247.32 \pm 29.32^{\mathrm{a}}$ \\
Final weight $(\mathrm{g})$ & $266.81 \pm 41.89^{\mathrm{a}}$ & $263.62 \pm 60.14^{\mathrm{a}}$ & $284.87 \pm 38.78^{\mathrm{a}}$ \\
$\begin{array}{c}\text { Feed uptake }(\mathrm{g} / \mathrm{d}) \text { per } \\
\text { animal }\end{array}$ & $19.02 \pm 1.47^{\mathrm{a}}$ & $13.18 \pm 7.40^{\mathrm{a}}$ & $21.76 \pm 3.82^{\mathrm{a}}$ \\
$\begin{array}{c}\text { Feed efficiency } \\
\text { Liver weight }\end{array}$ & $0.012 \pm 0.02^{\mathrm{a}}$ & $0.007 \pm 0.01^{\mathrm{a}}$ & $-0.095 \pm 0.09^{\mathrm{a}}$ \\
& $3.86 \pm 1.02^{\mathrm{a}}$ & $3.27 \pm 0.88^{\mathrm{a}}$ & $3.60 \pm 1.52^{\mathrm{a}}$ \\
\hline
\end{tabular}

Values in the same row with different roman superscripts are statistically significant $(\mathrm{P}<0.05)$. While values in the same row with the same roman superscript are insignificant $(P>0.05)(n=6)$. 
increased. When the concentration of saline was between $0.1 \%-0.35 \%$, insignificant difference was noted among the three diet regimes. At higher concentrations $(0.4 \%-0.85 \%)$, rats fed dietary oxidized oil showed significant higher level of hemolysis than their counterparts in the control group. Although vitamin $\mathrm{E}$ supplementation decreased hemolysis occurred at higher concentration, but this effect was statistically insignificant. Results of the current study are in contrast with results of Zhong et al. [7] who found that supplementation of dietary oxidized oil with $\alpha$-tocopherol seemed to decrease hemolysis of RBC of juvenile Atlantic cod Gadusmorhus. The EOF of erythrocytes was decreased by feeding oxidized oils to rats, which could be attributed to changes in their membranes, possibly due toincorporation of lipid oxidation products into erythrocytes membranes [1]. Thermally oxidized oil fed animals showed increased EOF especially at higher concentration, probably due to increase the oxidative stress on erythrocytes or could be due to antioxidant (vitamin E) deficiency. These two probable reasons need further study to investigate their mode of action. Addition of vitamin $\mathrm{E}$ to the diet containing highly oxidized oil resulted in lowering EOF, which lends further support to the latter explanation. Supplementation the diet with $\alpha$-tocopherol could enhance the resistance of RBC membranes to hemolysis. A survey of the scientific literature showed conflicting results regarding the damaging effects of thermally oxidized oils on gastric and intestinal muscosa. The role of vitamin $\mathrm{E}$ in rectifying this damage and, thus enhancing the iron absorption needs further studies.

When inspecting Figure 3, it showed that on day 90 post feeding, the level of serum TAG did not change significantly among animals fed oxidized oils compared with the control group. Against expectation, significant increase in TAG level was noted among rats fed oxidized oil and supplemented with vitamin $\mathrm{E}$ compared with their counter parts in the fresh and oxidized oil groups. Surprisingly, significant high level of TAG was noted among animals fed frying oil with vitamin E supplementation. These results contradict findings of Eder [1], who showed that dietary oxidized lipids decrease plasma levels of TAG. The significant increase in TAG level among animals fed oxidized oil and supplemented with the antioxidant ( $\alpha$-tocopherol) could be explained as the following. Antioxidants such as vitamin E might enhance the efficiency of pancreatic lipase. This is in accordance with literature reports [9].

Although serum TC levels were higher in diets containing oxidized oil with and without vitamin E supplementation than the control animals, but this difference was insignificant (Figure 4). Lipid chemistry study showed that feeding frying oil with or without supplementation of vitamin E had little effect on serum total cholesterol (TC). Results presented here disagreed with those of Shaeizadeh et al. [5] who showed that rats fed oxidized oil had higher levels of TC than their counterparts in the control group, or even among animals fed oxidized oil and fiber (pectin). Furthermore, there are also research studies reporting elevated TC in animals fed oxidized oils [1] [15]. For example, Eder [1] 


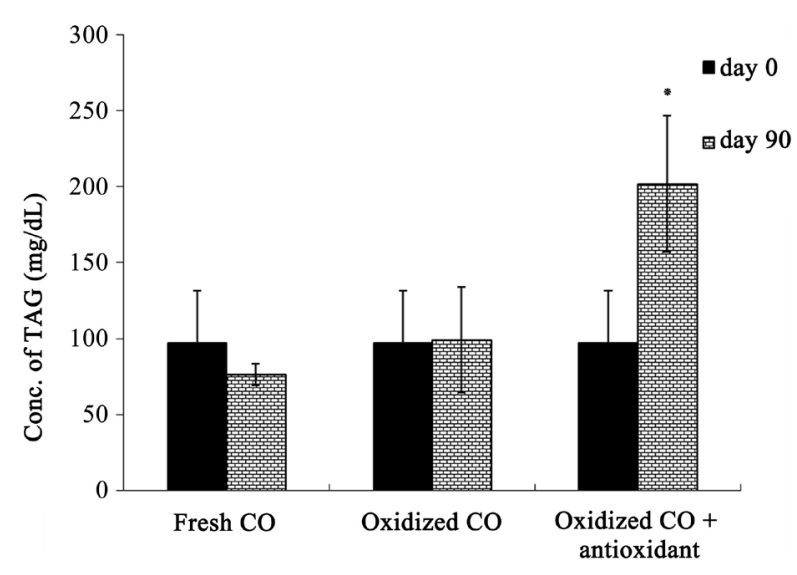

Figure 3. Triacylglycerol (TAG) level (mg/dL) for animals fed fresh corn oil, oxidized corn oil and oxidized corn oil with antioxidant at day zero and after 90 days of feeding. ${ }^{*} \mathrm{P}<0.05$ vs. fresh oil and oxidized oil. Data are the mean \pm standard deviation (SD) of six rats per each treatment.

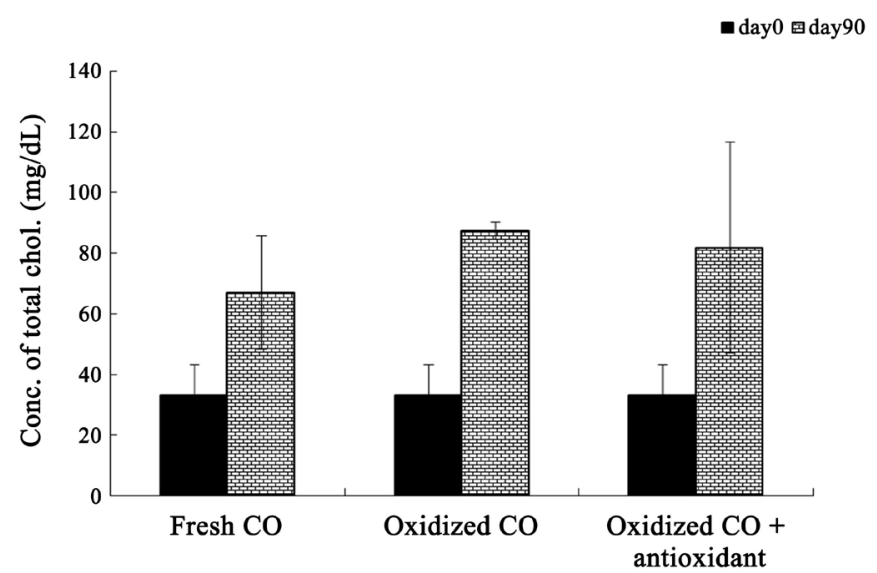

Figure 4. Total cholesterol (TC) level (mg/dL) for animals fed fresh corn oil, oxidized corn oil and oxidized corn oil with antioxidant at day zero and after 90 days of feeding. Error bars represents the standard deviation. Insignificant effect between the three treatments $(P>0.05)$ was noted. Data are the mean \pm standard deviation (SD) of six rats per each treatment.

found that rats fed oxidized oil with pectin had slightly lower level of plasma TC, possibly due to improved cholesterol fecal excretion. On contrary, Ammouche et al. [6] demonstrated a decrease in TC and TAG levels among animals fed oxidized sunflower oil compared to the fresh oil group. This decrease could be attributed to a reduction in TC absorption, possibly due to high amounts of oxidized esters. Another probable explanation for such decrease, might be due to a decrease in biosynthesis of cholesterol [6]. In the current study, increased TC amount in oxidized oil group could be due to enhanced reactivity of 5-hydroxy-3-methylglutaryl-coenzyme A reductase, which is in line with the literature [16] [17]. Increased activation of $\alpha$-hydrxylase, an enzyme accountable for cholesterol transformation in biliary acids might be another possible explanation. However, further studies are highly recommended to investigate the pre- 
viously mentioned explanation.

Even though vitamin E supplementation to the diet containing oxidized oil resulted in an increase in serum HDL-C level compared to the control group, but this increase was insignificant (Figure 5). Furthermore, results indicated a slight and insignificant increase in HDL-C level among animals fed only oxidized oil (without vitamin E supplementation) compared to animals fed fresh oil. Serum LDL-C level did not display any considerable difference among three treatments (Figure 6). Although the level of LDL-C of the vitamin E supplemented group looks like almost double of the non-supplemented group at day 90, but this difference was statistically insignificant. The same pattern was noted for serum HDL-C level, which disagreed with Shafeizadeh et al.

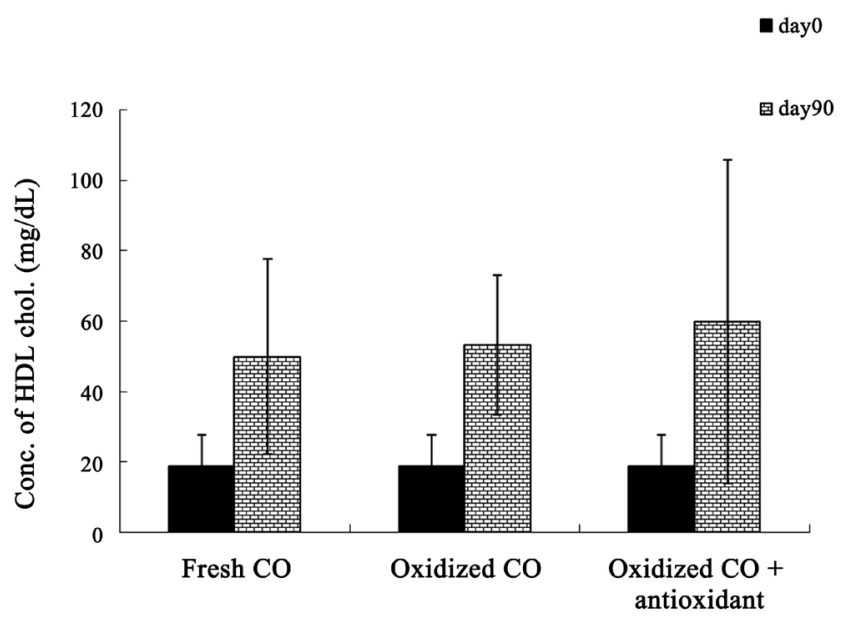

Figure 5. High-density lipoprotein (HDL-C) level (mg/dL) for rats fed fresh corn oil, oxidized corn oil and oxidized corn oil with antioxidant at day zero and after 90 days of feeding. Error bars represents the standard deviation. Data are the mean \pm standard deviation (SD) of six rats per each treatment.

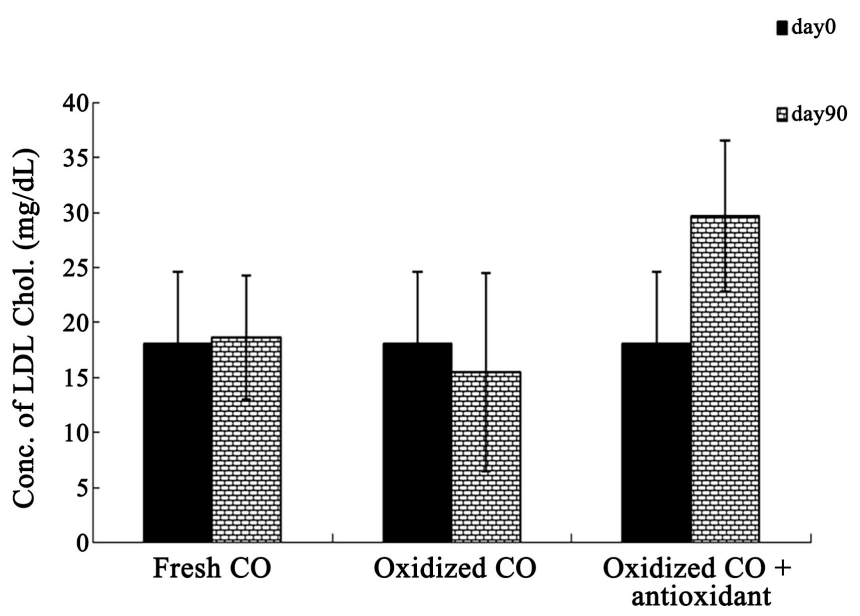

Figure 6. High-density lipoprotein (LDL-C) level (mg/dL) for rats fed fresh corn oil, oxidized corn oil and oxidized corn oil with antioxidant at day zero and after 90 days of feeding. Error bars represents the standard deviation. Data are the mean \pm standard deviation (SD) of six rats per each treatment. 
[5] demonstrated that rats fed dietary oxidized oil had considerably higher levels of HDL-C than other treatments "fresh oil", "fresh oil + pectin", and "oxidized oil + pectin". Shafeizadeh and his team [5] explained their results as the following: low uptake of linoleic acid, and a protective mechanism against the stress caused by oxidation. Additionally, results of the current study contradict findings of Falade et al. [18], who found that Wistar rats fed thermally oxidized plam oil for 30 days showed significant decrease in the plasma and liver levels of HDL-C and TAG.

It is worth to mention that results of this current study were based on short-term (90 days) impacts of oxidized oil and antioxidant on rats. Long-term feeding trial is highly recommended. Furthermore, determination of endogenous antioxidant enzymes, level of oxidation stress, and hydroperoxides and TABRS is also highly encouraged. This study did not investigate the plasma and liver function markers. It also did not determine fatty acid profile of plasma and liver. The selected dose of vitamin E might be in inadequate. Thus, further study is recommended to determine optimum vitamin $\mathrm{E}$ dosage.

\section{Conclusion}

Results of the present study suggested that the ingestion of frying oil with or without vitamin E supplementation had no significant effects on growth performance, blood lipid chemistry, and blood parameters. The only significant effect of frying oil was shown on erythrocytes hemolysis at higher concentration.

\section{Funding}

This study did not receive any funding from any sources.

\section{Author's Contributions}

FH played a central role in planning, desiring, and conducting this in vivo study. $\mathrm{He}$ also actively engaged in data collection and analysis. $\mathrm{FH}$ also contributed to writing the manuscript. AE helped in planning and executing the feeding study. He also contributed to writing manuscript.

\section{Conflict of Interests}

The authors of the present study declare that no competing interests.

\section{References}

[1] Eder, K. (1999) The Effects of a Dietary Oxidized Oil on Lipid Metabolism in Rats. Lipids, 34, 717-725. https://doi.org/10.1007/s11745-999-0418-0

[2] Hamam, F.A., Eldalo, A.S.E., Alnofeie, A.A., Alghamdi, W.Y., Almutairi, S.S. and Badyan, F.S. (2017) The Association of Eating Habits and Lifestyle with Overweight and Obesity among Health Sciences Students in Taif University, KSA. Journal of Taibah University and Medical Sciences, 12, 249-260.

https://doi.org/10.1016/j.jtumed.2016.12.001

[3] Hamam, F.A., Eldalo, A.S.E., Khaleel, M., Alwagdani, A., Alqarni, A., Daghas, B.M. 
A., Alharthy, H., Hassen, S., Alsofiany, A. and Alotaibie, S. (2017) Effects of Food Habits and Lifestyle on Prevalence of Overweight/Obesity among Schoolchildren in Taif Area, KSA. Food and Nutrition Sciences, 8, 196-211. https://doi.org/10.4236/fns.2017.82013

[4] Hamam, F. (2017) Dyslipidemia and Related Risk Factors in a Saudi University Community. Food and Nutrition Sciences, 8, 56-69. https://doi.org/10.4236/fns.2017.81004

[5] Shafaeizadeh, S., Jamalian, J., Owji, A.A., Azadbakht, L., Ramezani, R., Karbalaei, N., Rajaeifard, A. and Tabatabai, N. (2011) The Effect of Consuming Oxidized Oil Supplemented with Fiber on Lipid Profiles in Rat Model. Journal of Research and Medical Sciences, 16, 1541-1549.

[6] Ammouche, A., Rouaki, F. and Bellal, M.M. (2002) Effect of Ingestion of Thermally Oxidized Sunflower Oil on the Fatty Acid Composition and Antioxidant Enzymes of Rat Liver and Brain in Development. Annals of Nutrition and Metabolism, 46, 268-275. https://doi.org/10.1159/000066496

[7] Zhong,Y., Lall, S.P. and Shahidi, F. (2008) Effects of Dietary Oxidized Oil and Vitamin E on the Growth, Blood Parameters and Body Composition of Juvenile Atlantic Cod Gadus Morhua (Linnaeus 1758). Aquaculture Research, 39, 1647-1657.

[8] Yen, P.L., Chen, B.H., Yang, F.L. and Lu, Y.-F. (2010) Effects of Deep-Frying Oil on Blood Pressure and Oxidative Stress in Spontaneously Hypertensive and Normotensive Rats. Nutrition, 26, 331-336. https://doi.org/10.1016/j.nut.2009.04.020

[9] Olivero-David, R., Bastid, S., Fogliano, V., Vitaglione, P., Gonzalez-Muñoz, M.J., Sacchi, R., Sanchez-Muniz, F.J. and Benedi, J. (2010) Effect of Thermally Oxidized Oil and Fasting Status on the Short-Term Digestibility of Ketolinoleic Acids and Total Oxidized Fatty Acids in Rats. Journal of Agricultural and Food Chemistryy, 59, 4684-4691. https://doi.org/10.1021/jf1048063

[10] Aladedunye, F. and Przybylski, R. (2009) Degradation and Nutritional Quality Changes of Oil during Frying. Journal of the American Oil Chemists' Society, 86, 149-156. https://doi.org/10.1007/s11746-008-1328-5

[11] Sebastian, A., Ghazani, S.M. and Marangoni, A.G. (2014) Quality and Safety of Frying Oils Used in Restaurants. Food Research International, 64, 420-423. https://doi.org/10.1016/j.foodres.2014.07.033

[12] Friedewald, W.T., Levy, R.I. and Fredrickson, D.S. (1972) Estimation of the Concentration of Low-Density Lipoprotein Cholesterol in Plasma, without Use of the Preparative Ultracentrifuge. Clinical Chemistry, 18, 499-502.

[13] Mesembe, O.E., Ibanga, I. and Osim, E.E. (2004) The Effects of Fresh and Thermooxidized Palm Oil Diets on Some Haematological Indices in the Rat. Nigerian Journal of Physiological Sciences, 19, 86-91.

[14] Ani, E.J., Nna, V.U., Obi, C.E. and Udobong, N.J. (2015) Comparative Effects of Thermoxidized Palm Oil and Groundnut Oil Diets on Some Haematological Parameters in Albino Wistar Rats. Australian Journal of Basic and Applied Sciences, 9, 181-184.

[15] Borsting, C.F., Engberg, R.M., Jakobsen, K., Jensen, S.K. and Andersen, J.O. (1994) Inclusion of Oxidized Fish Oil in Mink Diets. The Influence on Nutrient Digestibility and Fatty-Acid Accumulation in Tissues. Journal of Animal Physiology and Animal Nutrition, 72, 132-45. https://doi.org/10.1111/j.1439-0396.1994.tb00380.x

[16] Sánchez-Muniz, F.J., López-Varela, S., Garrido-Polonio, M.C. and Cuesta, C. (1998) Dietary Effects on Growth, Liver Peroxides, and Serum and Lipoprotein Lipids in Rats Fed a Thermoxidised and Polymerised Sunflower Oil. Journal of Sciences and 
Food Agriculure, 76, 364-372.

https://doi.org/10.1002/(SICI)1097-0010(199803)76:3<364::AID-JSFA951>3.0.CO;2-E

[17] Hochgraf, E., Mokady, S. and Cogan, U. (1997) Dietary Oxidized Linoleic Acid Modifies Lipid Composition of Rat Liver Microsomes and Increases Their Fluidity. Journal of Nutrition, 127, 681-686. https://doi.org/10.1093/jn/127.5.681

[18] Falade, A.O., Oboh, G., Ademiluyi, A.O. and Odubanjo, O.V. (2015) Consumption of Thermally Oxidized Palm Oil Dietsalters Biochemical Indices in Rats. Beni-Suef University Journal of Basic and Applied Sciences, 4, 150-156.

https://doi.org/10.1016/j.bjbas.2015.05.009 